\title{
Characterization of time-resolved laser differential phase using 3D complementary cumulative distribution functions
}

\author{
Anthony J. Walsh, ${ }^{1,2, *}$ John A. O’Dowd, ${ }^{1,3,4}$ Vivian M. Bessler, ${ }^{1}$ Kai Shi, ${ }^{5}$ Frank Smyth, ${ }^{5}$ James M. Dailey, ${ }^{1,4}$ \\ Bryan Kelleher, ${ }^{1,6}$ Liam P. Barry, ${ }^{5}$ and Andrew D. Ellis ${ }^{1,4,5}$ \\ ${ }^{1}$ Tyndall National Institute, Lee Maltings, Cork, Ireland \\ ${ }^{2}$ Electrical and Electronic Engineering Department, University College Cork, Cork, Ireland \\ ${ }^{3}$ Intune Networks, Beckett Way, Parkwest, Dublin 12, Ireland \\ ${ }^{4}$ Physics Department, University College Cork, Cork, Ireland \\ ${ }^{5}$ The Rince Institute, Dublin City University, Glasnevin, Dublin 9, Ireland \\ ${ }^{6}$ Centre for Advanced Photonics and Process Analysis, Cork Institute of Technology, Bishopstown, Cork, Ireland \\ ${ }^{*}$ Corresponding author: anthony.walsh@tyndall.ie
}

Received February 6, 2012; revised March 31, 2012; accepted April 10, 2012;

posted April 10, 2012 (Doc. ID 162541); published May 15, 2012

\begin{abstract}
An experimental method for characterizing the time-resolved phase noise of a fast switching tunable laser is discussed. The method experimentally determines a complementary cumulative distribution function of the laser's differential phase as a function of time after a switching event. A time resolved bit error rate of differential quadrature phase shift keying formatted data, calculated using the phase noise measurements, was fitted to an experimental time-resolved bit error rate measurement using a field programmable gate array, finding a good agreement between the time-resolved bit error rates. () 2012 Optical Society of America

OCIS codes: $\quad 060.0060,060.1660,060.5060$.
\end{abstract}

It is projected that network traffic will increase by a factor of thirty in the next ten years [1], while communication resources are becoming scarce. Optical burst switching provides an opportunity to increase network resource utilization. In order to implement this, tunable lasers (TLs) should be able to switch among different wavelengths on the order of nanoseconds.

While moderately high information spectral densities may be achieved using direct detection, coherent detection is essential to maximize fiber capacity. It is therefore important to characterize the phase dynamics of fast TLs, which may simultaneously exhibit large laser frequency drift and variations in phase noise [2]. One method used to measure the phase noise of a continuous wave semiconductor laser is a delayed-self heterodyne technique [3]. This method was applied in real time but suffered from poor temporal resolution. A coherent receiver may be used to measure laser phase noise [4], and a carrier's frequency and phase may be separated by employing a full coherent transmitter and receiver pair [5] or using a novel coherent heterodyne receiver that has also been used to find the instantaneous linewidth [6]. Such methods typically attempt to separate deterministic frequency drifts from a random phase noise distributions (Wiener process) in order to predict system performance.

In this Letter, we demonstrate that such separation is unnecessary for accurate bit error rate (BER) predictions. This is achieved by characterizing the timeresolved differential phase of an unmodulated tunable laser under switching conditions using a coherent receiver. The acquired differential phase distributions contain all information pertaining to deterministic (frequency drift) and nondeterministic (phase noise, white noise, etc.) variations and allow direct BER prediction without inferring parameters such as frequency drift or linewidth. This technique makes no assumptions about the phase noise distribution or the frequency chirp profile, although the frequency chirp must be within the bandwidth of the measurement equipment.

Figure 1 shows the measurement setup. A sampled grating distributed Bragg reflector was used for the fast-switching tunable laser (FTL) and was switched periodically between two wavelengths by driving the rear grating section from a CG635 clock generator at $6.9 \mathrm{MHz}$ (the remaining sections were connected to dc current supplies). The FTL output was combined with a low linewidth external cavity laser (ECL) at a wavelength close to one of the FTL's channels using a $90^{\circ}$ optical hybrid. The four outputs were detected using balanced detectors and electrically sampled at $20 \mathrm{GSa} / \mathrm{s}$ using a real-time oscilloscope, with an analogue bandwidth of $10 \mathrm{GHz}$.

Assuming that the ECL phase noise (linewidth $133 \mathrm{kHz}$ ) is much less than that of the FTL, the observed instantaneous phase, $\phi_{\text {coh }}(t)$ will be given by

$$
\phi_{\mathrm{coh}}(\mathrm{t})=\Delta \omega(\mathrm{t}) \mathrm{t}+\phi_{\mathrm{ns}}(\mathrm{t})+\phi_{0},
$$

where $\Delta \omega(t)$ is the difference in angular frequency between the switching FTL and the ECL; $\phi_{\mathrm{ns}}(t)$ is the phase noise of the switching FTL; $\phi_{0}$ is the difference in initial phase of the two lasers, assumed to be quasi static over each measurement interval; and $t$ is the sampling time offset with respect to the start of the switching event.

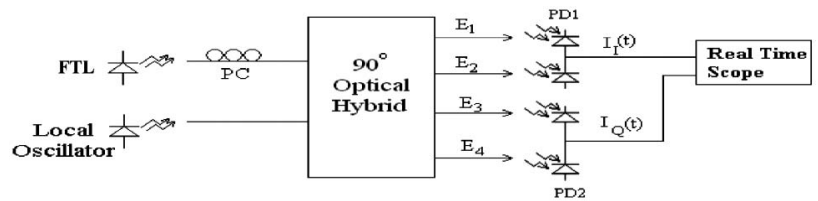

Fig. 1. Schematic diagram of coherent receiver setup to measure the instantaneous phase of a fast-switching tunable laser. 
Frequency chirp was expected, but it was not certain if phase noise distributions would vary with time. Conventional approaches separate $\Delta \omega(t)$ and assume particular statistics for $\phi_{\mathrm{ns}}(t)$. We analyze the instantaneous phase, $\phi_{\text {coh }}(t)$, directly.

In the experiment conducted, sets of 80 consecutive switching events were recorded separately by the realtime oscilloscope. To locate switching events within a set, timing markers were extracted when the amplitude of the $I$ channel from the coherent receiver dropped by approximately a factor of two to $10 \mathrm{mV}$. This enabled the switching events to be aligned, and receiver time skews, power imbalances, and offsets in $I$ and $Q$ were corrected using offline digital signal processing for each switching event. The $I$ and $Q$ outputs of the coherent receiver were used to acquire the instantaneous phase, $\phi_{\mathrm{coh}}(t)$. The differential phase, $\Delta \phi_{\text {coh }}(t)$, was calculated as the difference in instantaneous phase between two points in time:

$$
\Delta \phi_{\text {coh }}(\mathrm{t})=\phi_{\text {coh }}(\mathrm{t}-\mathrm{T})-\phi_{\text {coh }}(\mathrm{t}),
$$

where $T=2 T_{s}$, and $T_{s}$ is a measurement sampling period. $T$ will be referred to as the sampling interval ( $T=100 \mathrm{ps}$ in this work).

The distribution of the differential phase is calculated from 127 sets, each with 80 consecutive switching events $(10,160$ switching events in total), which were combined to produce histograms at different sampling times, $t$, between $-\pi$ and $\pi$ radians with a $\pi / 2000$ radians bin width. An example of one histogram, taken at $t=5.1 \mathrm{ns, \text {is }}$ shown in Fig. 2 as the red curve whose vertical axis is on the left. Note that in practice there was some frequency drift in the ECL between switching events. The steady-state difference in frequency between the FTL and the ECL was observed over the 50 ns to 65 ns time interval with respect to the start of each switching event for each switch. The range of the steady-state frequency difference among all the switching events was estimated to be $160 \mathrm{MHz}$. This error was not compensated for and will cause a negligible maximum error in the phase of 0.1 radians over 100 ps. A complementary cumulative distribution function (CCDF) (greater-than or equal-to type) of $\Delta \phi_{\text {coh }}(t)$ can be calculated from the differential phase histograms at different times, $t$. The CCDF at $5.1 \mathrm{~ns}$ is also shown in Fig. 2 as the black curve whose vertical axis is on the right.

A three-dimensional CCDF plot of the probability of exceeding a given differential phase may be calculated as shown in Fig. 3 , where color represents the probability

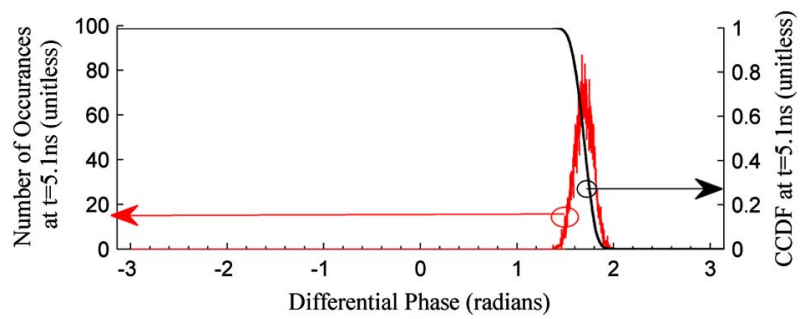

Fig. 2. (Color online) Histogram of differential phase (red with vertical axis on the left) and CCDF of differential phase (black with vertical axis on the right) at $5.1 \mathrm{~ns}$ with a sampling interval of 100 ps.

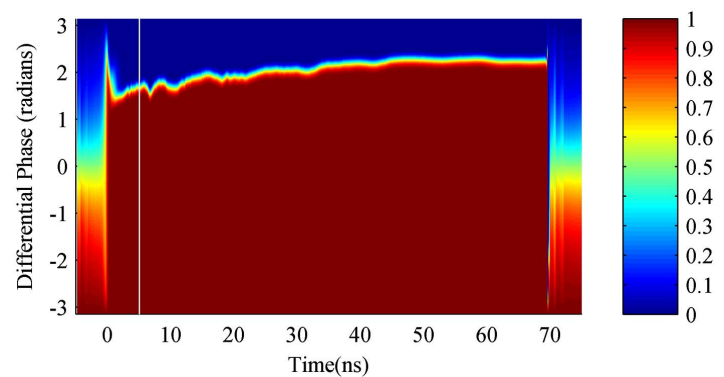

Fig. 3. (Color online) Time-resolved CCDF plotted for a sampling interval of $100 \mathrm{ps}$. White vertical line indicates CCDF from Fig. $\underline{2}$.

of the differential phase exceeding a particular differential phase ( $y$-axis) at a particular sampled time after the wavelength switch ( $x$-axis), plotted for a sampling interval of $100 \mathrm{ps}$. This profile fully describes the temporal evolution of the laser phase, including all variations originating from both linewidth and frequency chirp.

We next removed the frequency offset between the ECL and the FTL from the sampled phase evolution using an estimated frequency offset. This offset is calculated by finding the average of the slopes of the instantaneous phase between $50 \mathrm{~ns}$ and $65 \mathrm{~ns}$. Removing the offset from the instantaneous phase allows a "corrected instantaneous phase," $\phi_{\text {coh-cor }}(t)$, to be calculated from this value. Figure 4 shows an example time-resolved CCDF plot of the absolute value of the corrected differential phase $\left|\Delta \phi_{\text {coh-cor }}(t)\right|$.

In Fig. 4, the colors that are covered by the white horizontal line at $\pi / 4$ indicate the probability that the absolute corrected differential phase will be greater than or equal to $\pi / 4$ at different points in time. A similar statement can be made for the $3 \pi / 4$ white line. For hard decision differential quadrature phase shift keying (DQPSK), there will be a BER of 0.5 if the absolute corrected differential phase is between $\pi / 4$ and $3 \pi / 4$ and a BER of 1 if the absolute corrected differential phase is greater than $3 \pi / 4$. Hence, the BER of hard decision detected DQPSK at each point in time will be equal to

$$
\begin{aligned}
\mathrm{BER}= & \frac{1}{2} \mathrm{P}\left(\frac{3 \pi}{4}>\left|\Delta \phi_{\text {coh-cor }}(\mathrm{t})\right| \geq \frac{\pi}{4}\right) \\
& +\mathrm{P}\left(\left|\Delta \phi_{\text {coh-cor }}(\mathrm{t})\right| \geq \frac{3 \pi}{4}\right) .
\end{aligned}
$$

The data from Fig. 4 may thus be used to determine the time resolved BER (TR-BER) induced by the phase

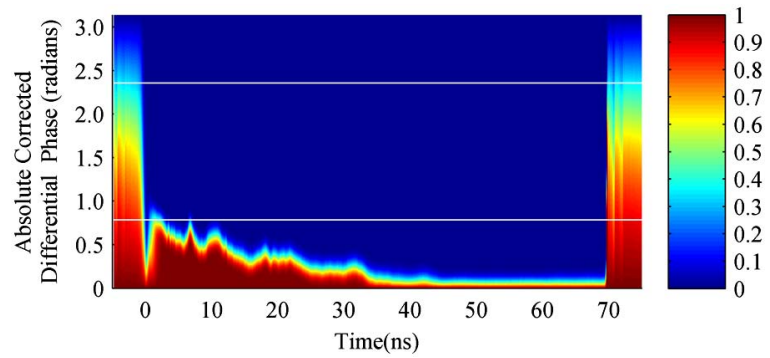

Fig. 4. (Color online) Time-resolved CCDF of the absolute corrected differential phase; the white horizontal lines represent the DQPSK tolerance lines at $\pi / 4$ and at $3 \pi / 4$. 


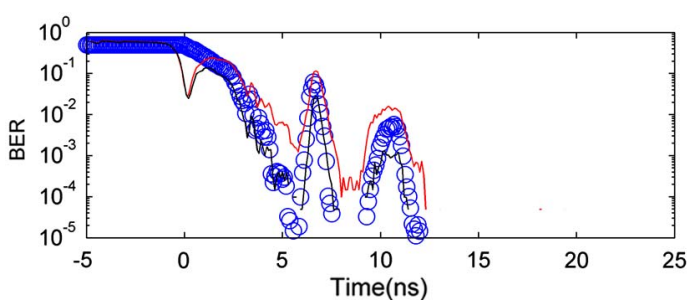

Fig. 5. (Color online) Plot of C-BERs (continuous red line with steady-state frequency removed and black line with AMZI bias removed) and experimentally acquired TR-BER (blue circles). Note that while the value of the sampling interval, $T$, is $100 \mathrm{ps}$ for the CCDFs, and the measured data has a symbol period of $93 \mathrm{ps}$, no correction for this difference was required.

variations for a given symbol interval arising from phase noise and frequency variations.

In order to confirm the accuracy of the calculated BER (C-BER) evolution, it was compared with an experimentally measured TR-BER. The measured data signal was generated using the same FTL modulated with a $2^{7}-1$ pseudo-random bit sequence from a standard pattern generator at 10 Gbaud by a dual parallel Mach-Zehnder modulator and assessed using a similar setup to that described in [7]. The signal was demodulated using an asymmetric Mach-Zehnder interferometer (AMZI) with a 1-bit delay and differentially detected using singleended detection. The AMZI was tuned to minimize the overall number of errors within the bursts. A fieldprogrammable gate array was used to compare the received data signal with the expected data signal. Note that the experimentally acquired TR-BER and the timeresolved laser instantaneous phase measurements were done simultaneously. Differential detection was used to guarantee independence from the coherent receiver measurements used to calculate the instantaneous phase. High probability of errors occur near the start of the burst because of the mismatch between the FTL frequency and the frequency of the 1-bit delay interferometer, and impact of the stochastic phase noise, which has a greater impact when the frequency mismatch, is large.

To complete the comparison, it was necessary to further account for a slight frequency offset between the FTL's steady-state frequency and the transfer function of the AMZI. In practice, this "AMZI bias" was accounted for by adjusting the relative frequency of the FTL to improve the fit between the experimentally determined TR-BER and the C-BER. Figure 5 compares the C-BER with (black line) and without (red line) this AMZI bias correction to the experimentally measured TR-BER (blue circles). In addition to optimizing the AMZI bias, the timing offset between the C-BER and the TR-BER data was also matched. It can be seen that an excellent agreement between the calculated and experimental data is achieved when the AMZI bias offset is taken into account. All CBER calculations were carried out using $10^{-4}$ bursts to reduce the uncertainty in the predicted error rate. The experimental TR-BER settles to less than $10^{-9}$ after 14 ns. Small deviations near $0 \mathrm{~ns}$ and 10.5 ns exist between the experimental and C-BER (with AMZI bias correction), which we believe may result from the fact that the C-BER does not take output power fluctuations and frequency deviations beyond $10 \mathrm{GHz}$ into account. However, such features could readily be taken into account to increase accuracy. Note that without the AMZI bias offset correction, the C-BER overestimates the BER by up to one order of magnitude in regions of low experimentally observed TR-BER. If a coherent receiver were used, it would give a lower BER than a directly detected system.

In conclusion, a technique for characterizing timeresolved laser differential phase has been presented. This technique determines a CCDF of the differential phase referred to a specific sampling interval as a function of time after a switching event and operates independently of the laser phase noise distribution or frequency chirp profile. Using our technique, we were able to accurately fit the C-BER of a DQPSK signal to an experimentally measured TR-BER.

This work was supported by Science Foundation Ireland under grant numbers 09/IN.1/I2653, 07/SRC/I117, 06/ IN/I969, and 07/IN.1/1929, and by Enterprise Ireland under grant number CFTD/08/333. The authors would like to thank Colm O'Riordan for useful discussions.

\section{References}

1. D. J. Bishop, A. R. Hartman, D. C. Kilper, S. K. Korotky, and D. Suvakovic, in Military Communications Conference, 2010 (Milcom, 2010), pp. 2047-2049.

2. A. K. Mishra, A. D. Ellis, L. P. Barry, and T. Farrell, in Optical Fiber Communication Conference and Exposition and The National Fiber Optic Engineers Conference (Optical Society of America, 2008), paper OTuC4.

3. D. Derickson, Fibre Optic Test and Measurement (Prentice Hall, 1998).

4. K. Kikuchi, Opt. Express 20, 5291 (2012).

5. B. C. Thomsen, R. Maher, D. S. Millar, and S. J. Savory, Opt. Express 19, B770 (2011).

6. K. Shi, R. Watts, D. Reid, T. N. Huynh, C. Browning, P. M. Anandarajah, F. Smyth, and L. P. Barry, IEEE Photon. Technol. Lett. 23, 1591 (2011).

7. J. A. O'Dowd, V. M. Bessler, S. K. Ibrahim, A. J. Walsh, F. H. Peters, B. Corbett, B. Roycroft, P. O. Brien, and A. D. Ellis, in International Conference on Transparent Optical Networks 2011 (IEEE, 2011), paper We.D1.2. 